\title{
Not the Typical Pneumonia: An Unusual Case of Rasburicase-induced Methomoglobinemia
}

\author{
Moustafa Younis ${ }^{1}$, Laith Derbas ${ }^{2}$, Stephen M. Eikermann ${ }^{3}$, Majdi S. Hamarshi ${ }^{4}$ \\ 1. Internal Medicine, University of Missouri/St. Luke's Health System, Kansas City, USA 2. Internal Medicine, University \\ of Missouri, Kansas City, USA 3. Critical Care, University of Missouri/St. Luke's Health System, Kansas City, USA 4. \\ Critical Care, St Luke's Health System, Kansas City, USA
}

Corresponding author: Moustafa Younis, mustafais1@hotmail.com

\begin{abstract}
We present a rare case of rasburicase-induced methemoglobinemia and hemolytic anemia in the setting of presumed glucose-6-phosphate dehydrogenase (G6PD) deficiency. A 78-year-old male with a known history of chronic lymphocytic leukemia presented to the clinic with fever of unknown origin. Laboratory results were significant for hyperuricemia. He was empirically started on levofloxacin and rasburicase. He then presented to the emergency department with shortness of breath and syncope. Physical examination was remarkable for a fever of $102.8^{\circ} \mathrm{F}$, conjunctival pallor, and scleral icterus. An infiltrate was observed on his computed tomography (CT) angiogram of the chest. Arterial blood gas on $50 \%$ fraction of inspired oxygen was significant for an arterial oxygen level of 222 millimeters mercury and oxyhemoglobin of $85.9 \%$. Cooximetry was then obtained and methemoglobin level was $13.4 \%$. Laboratory results were noteworthy for a drop-in hemoglobin, indirect hyperbilirubinemia, low haptoglobin and elevated lactate dehydrogenase; depicting hemolytic anemia. The patient received two units of packed red blood cells, intravenous broadspectrum antibiotics and he clinically improved.
\end{abstract}

Categories: Internal Medicine, Oncology, Pulmonology

Keywords: methemoglobinemia, rasburicase, hemolysis, glucose-6-phosphate dehydrogenase deficiency

\section{Introduction}

Tumor lysis syndrome (TLS) is an oncological emergency referring to the constellation of metabolic disturbances resulting from the rapid destruction of tumor cells leading to the release of intracellular ions and nucleic acids, eventually metabolized to uric acid, into the blood stream [1]. The metabolic disturbances associated with TLS include hyperuricemia, hyperkalemia, hyperphosphatemia, hypocalcemia, and acute kidney injury (AKI) [2]. TLS can be precipitated either following the administration of cytotoxic chemotherapeutic agents or spontaneously in the setting of hematological malignancies [3].

Received 07/05/2018

Review began 07/12/2018 Review ended 07/22/2018 Published 08/01/2018

\section{(C) Copyright 2018}

Younis et al. This is an open access article distributed under the terms of the Creative Commons Attribution License CC-BY 3.0., which permits unrestricted use, distribution, and reproduction in any medium, provided the original author and source are credited.
In TLS, the main prophylactic methods include intravenous hydration, allopurinol and rasburicase administration [4]. For patients at high risk for TLS or in those where allopurinol is contraindicated, rasburicase is currently recommended by the Guidelines for the Management of Pediatric and Adult Tumor lysis syndrome published by the American Society of Clinical Oncology in 2008 [4]. Rasburicase is a recombinant urate-oxidase enzyme approved by the Food and Drug Administration (FDA) for the treatment and/or prevention of hyperuricemia and TLS. It converts uric acid to allantoin (an inactive and soluble metabolite of uric acid) facilitating the renal clearance of uric acid and decreasing its precipitation in the kidneys [5]. Multiple studies demonstrated a greater reduction in uric acid levels with a lower risk of AKI, compared to allopurinol [5-7].

Rasburicase is usually well tolerated; however, several side effects are of concern. Methemoglobinemia is an infrequent $(<1 \%)$ but serious complication associated with the administration of rasburicase [8]. In addition, the drug is contraindicated in patients with glucose-6-phosphate dehydrogenase (G6PD) deficiency as hydrogen peroxide (a by-product of uric acid catabolism) can present an oxidative stress thus, precipitating hemolysis in this setting [9]. There are United States (US) boxed warnings for both these side effects and advice to screen all African, South-East Asian, Middle Eastern, and Mediterranean patients for G6PD prior to rasburicase administration [10]. In the following, we illustrate the morbidity and management of rasburicase-induced methemoglobinemia and hemolysis in an African-American male patient with presumed G6PD.

\section{Case Presentation}

A 78-year-old African-American man weighing 53 kilograms with a past medical history significant for chronic lymphocytic leukemia (CLL), hyperuricemia with chronic gouty arthropathy, chronic kidney disease (CKD) stage 3 presented to the Emergency Department (ED) with the chief complaint of shortness of breath and fatigue for one day. His outpatient medication included ibrutinib 420 milligrams (mg) and allopurinol $300 \mathrm{mg}$ daily. He was diagnosed with CLL two years prior to presentation. Initially, he was started on bendamustine with an appropriate response. However, an escalation in his lymphocyte count was 


\section{Cureus}

appreciated one month prior to presentation and ibrutinib was initiated for CLL progression. One day prior to admission to the hospital, the patient was evaluated by his primary oncologist at an outpatient visit. He was febrile with a temperature of 101.6 degrees fahrenheit $\left({ }^{\circ} \mathrm{F}\right)$ but otherwise asymptomatic. Blood and urine cultures were obtained and was started on oral levofloxacin empirically. His labs were significant for worsening hyperuricemia with a uric acid level of 13.0 milligram per deciliter (mg/dl). A single dose of intravenous rasburicase $3 \mathrm{mg}$ was then administered.The following day, he presented to the ED complaining of significant fatigue associated with dry nonproductive cough of one day duration. Examination was remarkable for a fever of $102.8^{\circ} \mathrm{F}$, an oxygen saturation (SPO2) of $85 \%$ on room air, conjunctival pallor and scleral icterus. A left lower lobe infiltrate was observed on his computed tomography (CT) angiogram of the chest (Figure 1). Laboratory results were noteworthy for a drop-in hemoglobin $(4.9 \mathrm{mg} / \mathrm{dL})$, indirect hyperbilirubinemia (7.2 mg/dL), low haptoglobin ( $<10 \mathrm{mg} / \mathrm{dL})$ and elevated lactate dehydrogenase of 1611 International Units per liter (IU/L) compared to $(756 \mathrm{IU} / \mathrm{L})$ the day before; depicting acute hemolytic anemia. SPO2 persisted at $85 \%$ despite the use of nasal cannula, nonrebreather and non-invasive positive pressure ventilation. The patient was admitted to the intensive care unit (ICU). Arterial blood gas on $50 \%$ fraction of inspired oxygen (FiO2) was significant for an arterial oxygen level (PO2) of 222 millimeters mercury (mm $\mathrm{Hg}$ ) and an oxyhemoglobin of $85.9 \%$. Co-oximetry was then obtained and methemoglobin level was $13.4 \%$. The patient was started on vancomycin and cefepime and transfused with three units of packed red blood cells. Methylene blue was not administered as the patient was assumed to have G6PD deficiency given his race and this episode of hemolysis.

After an ICU stay of two days, the patient clinically improved, SpO2 normalized, his hemoglobin levels improved, methemoglobin levels trended down and was transferred back to the floor (Table 1). A G6PD level sent during the acute attack yielded a result within the normal range. In addition, mycoplasma serum antibodies were negative. Despite broad spectrum antibiotic coverage, the patient continued to spike fevers as high as $102.7^{\circ} \mathrm{F}$ from day one to day four. He subsequently started complaining of right knee pain associated with right knee swelling and tenderness on examination. Arthrocentesis revealed monosodium urate crystals and was started on colchicine and prednisone. Fever, knee pain, swelling and tenderness resolved. The patient was discharged to a skilled nursing facility on prednisone taper, allopurinol and colchicine. He was continued on ibrutinib to date (five months later) and his white blood cell count is currently within normal limits. A repeat CT chest performed three monthts later documented resolution of the previously seen left lower lobe pulmonary inifiltrate.

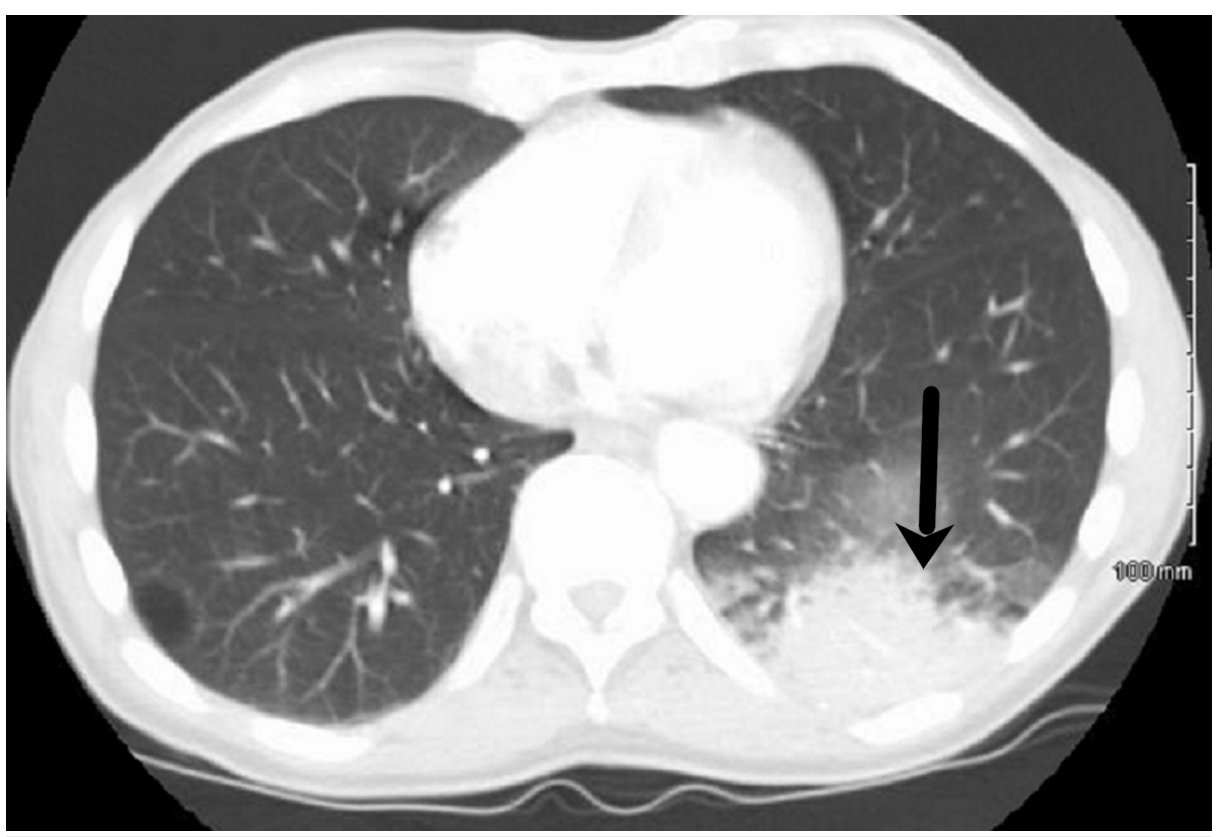

FIGURE 1: Computed tomography (CT) of the chest demonstrating a left lower lobe infiltrate 


\begin{tabular}{|c|c|c|c|c|c|c|c|c|c|}
\hline Value & Normal range & Day 0* & Day 1 & Day 2 & Day 3 & Day 4 & Day 5 & Day 6 & Day 7 \\
\hline Methemoglobin & $0-1.5 \%$ & NA & 13.4 & 4.4 & NA & 1.4 & NA & NA & NA \\
\hline Hemoglobin & $13-17 \mathrm{~g} / \mathrm{dl}$ & 8.3 & 4.9 & 8.0 & 8.1 & 8.6 & 8.2 & 7.9 & 7.9 \\
\hline Oxygen saturation & $95-100 \%$ & 100 & $75-88$ & $92-100$ & $98-100$ & $99-100$ & $99-100$ & $99-100$ & $99-100$ \\
\hline Lactate dehydrogenase & 313-618 IU/L & 756 & 1611 & NA & 2354 & 1713 & 1428 & 1224 & 1007 \\
\hline Total bilirubin & $0.2-1.3 \mathrm{mg} / \mathrm{dl}$ & 2.2 & 7.2 & 8.6 & 8.4 & 4.7 & 2.0 & 1.7 & 1.5 \\
\hline Uric acid & $2.5-7.0 \mathrm{mg} / \mathrm{dl}$ & 12.9 & 5.7 & 3.9 & 4.5 & 4.6 & 4.4 & 4.9 & 4.8 \\
\hline
\end{tabular}

\section{TABLE 1: The patient's clinical and laboratory values}

* Day 0 is clinic day (day prior to admission).

NA: not available; g/dl: grams per deciliter; IU/L: international units per liter; mg/dl: milligrams per deciliter.

\section{Discussion}

We present a rare case of rasburicase-induced methemoglobinemia and hemolytic anemia in the setting of G6PD deficiency. According to an international TLS expert consensus panel developed by Cairo et al., our patient was at high risk for developing TLS confirming the appropriate use of rasburicase in this scenario [11]. As previously mentioned, there is a US boxed warning advising to screen all African, South-East Asian, Middle Eastern and Mediterranean patients for G6PD prior to rasburicase administration [10]. Due to the urgency of the situation and the patient's worsening uric acid levels, despite being on allopurinol, our patient was not screened for G6PD prior to administration. The benefits of treating this oncological emergency outweighed the risks of administering rasburicase in a patient whose G6PD status is unknown. An important aspect in testing G6PD enzymatic activity is the timing. During acute hemolysis, the RBCs with severely reduced G6PD activity will hemolyze. So, the measured assay will reflect the activity of G6PD in the remaining $\mathrm{RBCs}$ which carry enzymes with a relatively normal activity, resulting in a false negative result.

Methemoglobinemia occurs when the ferrous ions incorporated in hemoglobin are oxidized to ferric ions [12]. Ferric ions are unable to reversibly bind to oxygen; thus, increasing the affinity of the remaining ferrous hemoglobin to oxygen, shifting the oxygen dissociation curve to the left and therefore decreasing the release of oxygen to the tissue inducing tissue hypoxia [12]. One of the cellular reactions involved in reducing methemoglobin to hemoglobin in the body utilizes nicotinamide adenine dinucleotide phosphate (NADPH) generated by the G6PD in the hexose monophosphate shunt. This reaction is not physiologically functional and requires activation by electron carriers such as methylene blue or riboflavin [13]. Therefore, the first line of therapy for the management of methemoglobinemia, in addition to avoiding the precipitating chemical, involves the use of methylene blue to facilitate the reduction of ferric to ferrous ions [14]. In patients with G6PD deficiency, NADPH generation is reduced thus this reaction is deemed useless and methylene blue is ineffective in such patients. Furthermore, methylene blue has oxidant potential which may induce hemolysis in G6PD deficient patients [15]. In these patients, ascorbic acid is recommended [16]. Ascorbic acid is slow in onset and requires up to 24 hours and therefore is a poor choice in such emergencies [16]. Reviewing the literature, only one case was successfully managed using ascorbic acid alone [9]. Other alternative therapies include simple or exchange blood transfusions and/or hyperbaric oxygen therapy. Given the combination of hemolysis and mild methemoglobinemia $<20 \%$ in our patient, we opted for a simple blood transfusion.

As per our knowledge, the number of rasburicase-induced methemoglobinemia reported cases is limited. Having both methemoglobinemia and acute hemolysis makes this case exceedingly rare. We suggest that simple transfusion alone is an effective alternative in the management of rasburicase-induced methemoglobinemia and hemolytic anemia, particularly in mild to moderate cases with methemoglobin levels less than $20 \%$. In such scenarios, simple transfusion serves two purposes: correcting the anemia and counteracting methemoglobinemia by supplying normal hemoglobin species preventing tissue hypoxia.

(Abstract: Younis M, Eikermann SM, Hamarshi MS. Not the Typical Pneumonia; an Unusual Case of Rasburicase Induced Methomoglobinemia. American Thoracic Society conference; May 18-23, 2018). https://www.atsjournals.org/doi/abs/10.1164/ajrccm-conference.2018.197.1_MeetingAbstracts.A6921

\section{Conclusions}

Rasburicase-induced methemoglobinemia and hemolytic anemia have been identified as a direct cause for 
morbidity and mortality in high-risk individuals. Methylene blue is strongly discouraged for rasburicaseinduced methemoglobinemia even if the G6PD status is unknown. Management with simple blood transfusions in mild to moderate severity cases is a relatively safe and effective strategy. Spreading awareness and using order sets to remind physicians of risks associated with rasburicase and methylene blue use might be helpful. It is enouraged to report such cases and highlight the management to help set up the stage to formulate a unified consensus for treatment.

\section{Additional Information}

\section{Disclosures}

Human subjects: Consent was obtained by all participants in this study. Conflicts of interest: In compliance with the ICMJE uniform disclosure form, all authors declare the following: Payment/services info: All authors have declared that no financial support was received from any organization for the submitted work. Financial relationships: All authors have declared that they have no financial relationships at present or within the previous three years with any organizations that might have an interest in the submitted work. Other relationships: All authors have declared that there are no other relationships or activities that could appear to have influenced the submitted work.

\section{References}

1. Howard SC: Tumor Lysis Syndrome. Abeloff's Clinical Oncology. Niederhuber JE (ed): Elsevier, Philadelphia; 2014. 41:591-596.

2. Hande KR, Garrow GC: Acute tumor lysis syndrome in patients with high-grade non-Hodgkin's lymphoma . Am J Med. 1993, 94:133-139.

3. Wössman W, Schrappe M, Meyer U, Zimmermann M, Reiter A: Incidence of tumor lysis syndrome in children with advanced stage Burkitt's lymphoma/leukemia before and after introduction of prophylactic use of urate oxidase. Ann Hematol. 2003, 82:160.

4. Coiffier B, Altman A, Pui CH, Younes A, Cairo MS: Guidelines for the management of pediatric and adult tumor lysis syndrome: an evidence-based review. J Clin Oncol. 2008, 26:2767-2678. 10.1200/JCO.2007.15.0177

5. Bosly A, Sonet A, Pinkerton CR, et al.: Rasburicase (recombinant urate oxidase) for the management of hyperuricemia in patients with cancer: report of an international compassionate use study. Cancer. 2003, 98:1048-1054. 10.1002/cncr.11612

6. Pui CH, Mahmoud HH, Wiley JM, et al.: Recombinant urate oxidase for the prophylaxis or treatment of hyperuricemia in patients with leukemia or lymphoma. J Clin Oncol. 2001, 19:697-704.

7. Jeha S, Kantarjian H, Irwin D, et al.: Efficacy and safety of rasburicase, a recombinant urate oxidase (Elitek), in the management of malignancy-associated hyperuricemia in pediatric and adult patients: final results of a multicenter compassionate use trial. Leukemia. 2005, 19:34-38. 10.1038/sj.leu.2403566

8. $\quad$ ELITEK ${ }^{\circledR}$ Prescribing Information. (2015). Accessed: January 6, 2018: http://products.sanofi.us/elitek/elitek.html.

9. Sonbol MB, Yadav H, Vaidya R, Rana V, Witzig TE: Methemoglobinemia and hemolysis in a patient with G6PD deficiency treated with rasburicase. Am J Hematol. 2013, 88:152-154.

10. FDA/Center for Drug Evaluation and Research OoC. Highlights of Prescribing Information . (2009). Accessed: January 5, 2018: https://www.accessdata.fda.gov/drugsatfda_docs/label/2009/103946s5083lbl.pdf.

11. Cairo MS, Coiffier B, Reiter A, Younes A: Recommendations for the evaluation of risk and prophylaxis of tumor lysis syndrome (TLS) in adults and children with malignant diseases: an expert TLS panel consensus. Br J Haematol. 2010, 149:578-586. 10.1111/j.1365-2141.2010.08143.x

12. Darling RC, Roughton FJW: The effect of methemoglobin on the equilibrium between oxygen and hemoglobin. Am J Physiol. 1942, 137:56-58.

13. Yubisui T, Takeshita M, Yoneyama Y: Reduction of methemoglobin through flavin at the physiological concentration by NADPH-flavin reductase of human erythrocytes. J Biochem. 1980, 87:1715-1720.

14. Benz EJ Jr, Silberstein LE, Heslop HE, Weitz JI, Anastasi J: Hematology: Basic Principles and Practice. Churchill Livingstone, Philadelphia, PA; 2013.

15. Reading NS, Ruiz-Bonilla JA, Christensen RD, Cáceres-Perkins W, Prchal JT: A patient with both methemoglobinemia and G6PD deficiency: a therapeutic conundrum. Am J Hematol. 2017, 92:474-477. 10.1002/ajh.24683

16. Rino PB, Scolnik D, Fustiñana A, Mitelpunkt A, Glatstein M: Ascorbic acid for the treatment of methemoglobinemia: the experience of a large tertiary care pediatric hospital. Am J Ther. 2014, 21:240-243. 10.1097/MJT.0000000000000028 